Cinémas

Revue d'études cinématographiques

Journal of Film Studies

\title{
Sois bonne, ô ma belle inconnue... (sur ritournelle et galop dans La Chienne)
}

\section{Jean-Louis Leutrat}

Volume 3, numéro 1, automne 1992

Cinéma et Musicalité

URI : https://id.erudit.org/iderudit/1001176ar

DOI : https://doi.org/10.7202/1001176ar

Aller au sommaire du numéro

Éditeur(s)

Cinémas

ISSN

1181-6945 (imprimé)

1705-6500 (numérique)

Découvrir la revue

Citer cet article

Leutrat, J.-L. (1992). Sois bonne, ô ma belle inconnue... (sur ritournelle et galop dans La Chienne). Cinémas, 3(1), 19-24. https://doi.org/10.7202/1001176ar
Résumé de l'article

À partir de deux concepts de Gilles Deleuze, « galop » et « ritournelle ", l'auteur rend compte ici du rôle de la chanson "Sois bonne, ô ma belle inconnue... " dans le film La Chienne de Jean Renoir. 


\title{
Sois bonne, ô ma belle inconnue... (sur ritournelle et galop dans La Chienne)
}

\section{Jean-Louis Leutrat}

\begin{abstract}
RÉSUMÉ
À partir de deux concepts de Gilles Deleuze, «galop» et «ritournelle», l'auteur rend compte ici du rôle de la chanson «Sois bonne, ô ma belle inconnue...» dans le film $\mathrm{La}$ Chienne de Jean Renoir.
\end{abstract}

\begin{abstract}
Taking as a starting point two concepts used by Gilles Deleuze, "galop" and "ritournclle", the author discusses the role of the song "Sois bonne, $\hat{o}$ ma belle inconnue..." in the film La Chienne by Jean Renoir.
\end{abstract}

Jean Renoir, qui aime beaucoup les chansons des rues, a déclaré qu'il avait peut-être tourné La Chienne pour la chanson d'Eugénie Buffet, qui accompagne le meurtre de Lulu (et qu'il reprend plus de vingt ans après dans French Cancan). Comme ce meurtre n'est pas montré, on est amené à penser que l'événement important, c'est la chanson elle-même dont l'utilisation, selon Michel Chion, n'est que le cas particulier d'une loi générale :

En choisissant d'inscrire un événement humain dans la durée fatale, promise à un proche arrêt, de sa petite forme toute découpée déjà, en marquant ineffaçablement le moment où elle commence et celui où elle finit, la musique peut prendre et isoler dans sa bulle un fragment court du temps de l'action, de ce temps réel, si lourd, du cinéma parlant, pour le porter au rang de destin (p. 132). 
Que représente dans un film une petite chanson? «Une forme fermée, répétitive, ponctuée par des cadences, des répétitions textuelles, des conclusions et des repos obligés» (Chion, p. 132). Non seulement la durée de la scène coïncide avec le déroulement de la complainte, mais la forme fermée et répétitive de cette dernière constitue à l'intérieur de la continuité du film un noyau intensément dramatique, assimilé étrangement à la légèreté d'une bulle dans le temps «lourd» de l'action. La chanson des rues relève de l'une des deux formes musicales propres au cinéma, selon Gilles Deleuze : la ritournelle, «forme a priori du temps qui fabrique chaque fois des temps différents» (Deleuze et Guattari, p. 431). La ritournelle est un «cristal d'espace-temps». Michel Chion retient l'idée du reflet lorsqu'il écrit :

La forme musicale la plus brève - telle qu'une chanson avec couplet et refrain - peut ainsi contracter, dans un laps très court, une série de scansions, de répétitions, de lois d'enchaînements, la figure d'un destin, la courbe d'une histoire et peut intervenir, dans les films, comme ces petites boules de verre rondes qu'on tient dans la main au milieu d'un pièce et qui la reflètent toute (pp. 132-133).

Deleuze retient les «vibrations variées, les décompositions, projections et transformations». Aussi choisit-il le prisme plutôt que la sphère. À propos de la scène de La Chienne, Jean-Louis Schefer parle d'«un corps de diamant» (p. 153).

Par ses paroles, en effet, la complainte d'Eugénie Buffet ouvre sur plusieurs moments du temps et sur plusieurs temporalités. La supplique «Sois bonne, ô ma belle inconnue» renvoie à la situation présente, alors que celle qui la prolonge, «Fais-moi la charité», annonce la fin du film, la clochardisation de Legrand vivant des aumônes qu'on lui fait. L'ambiguïté porte évidemment sur la nature de l'acte de charité : s'agit-il de l'argent (que convoite Lulu et que ramasse le chanteur dans la rue) ou de l'affection (que réclame Legrand)? La phrase par laquelle débute chaque couplet : «Si je passe sous ta fenêtre» évoque une situation qui s'est répétée dans le passé, mais aussi une image qui échappe à l'événementiel. La complainte revêt diverses significations selon le moment où on l'entend. Elle intervient dès que Legrand prend conscience qu'il a été dupé. Elle cesse après le départ du personnage. Si le chanteur s'arrête alors, la violoniste et le guitariste poursuivent et accompagnent la venue de Dédé jusqu'à ce que lui aussi quitte la scène. Tout de suite, une relation s'établit entre les paroles de la chanson ( $\mathrm{Si}$ je passe sous ta fenêtre / Ainsi qu'un galant troubadour...») et le dialogue qui s'échange dans la chambre et qui tourne vite aux insultes («Regarde-toi dans la glace», «saleté», «chienne», etc.). Cette relation est d'opposition et de contraste. 
Ensuite, le rire de Lulu et l'imploration de Legrand recouvrent la chanson. Ils instaurent, par-dessus la ritournelle, le rythme d'un galop qui va crescendo. Le galop est l'autre forme musicale propre au cinéma, selon Gilles Deleuze - «La précipitation des présents qui passent» (p. 123). Le rythme galopant est donné par les sons qui sortent de ces corps eux-mêmes saisis comme dans une étreinte, un corps à corps.

Le galop et la ritournelle, e'est ce qu'on entend dans le cristal, comme les deux dimensions du temps musical (...). Pour Renoir, la force de vie est du côté des présents qui s'élancent vers l'avenir, du côté du galop (...), tandis que la ritournelle a la mélancolie de ce qui retombe déjà dans le passé (Deleuze, p. 123).

Cela est vrai dans la mesure où le rythme entraîne un rapprochement physique des deux personnages qui transforme la scène du meurtre en une scène d'amour, et où, dans le même temps, la chanson parle d'une situation à jamais révolue. On peut dire aussi que le galop précipite une fin et que la ritournelle «soustrait au temps qui passe» un commencement du monde.

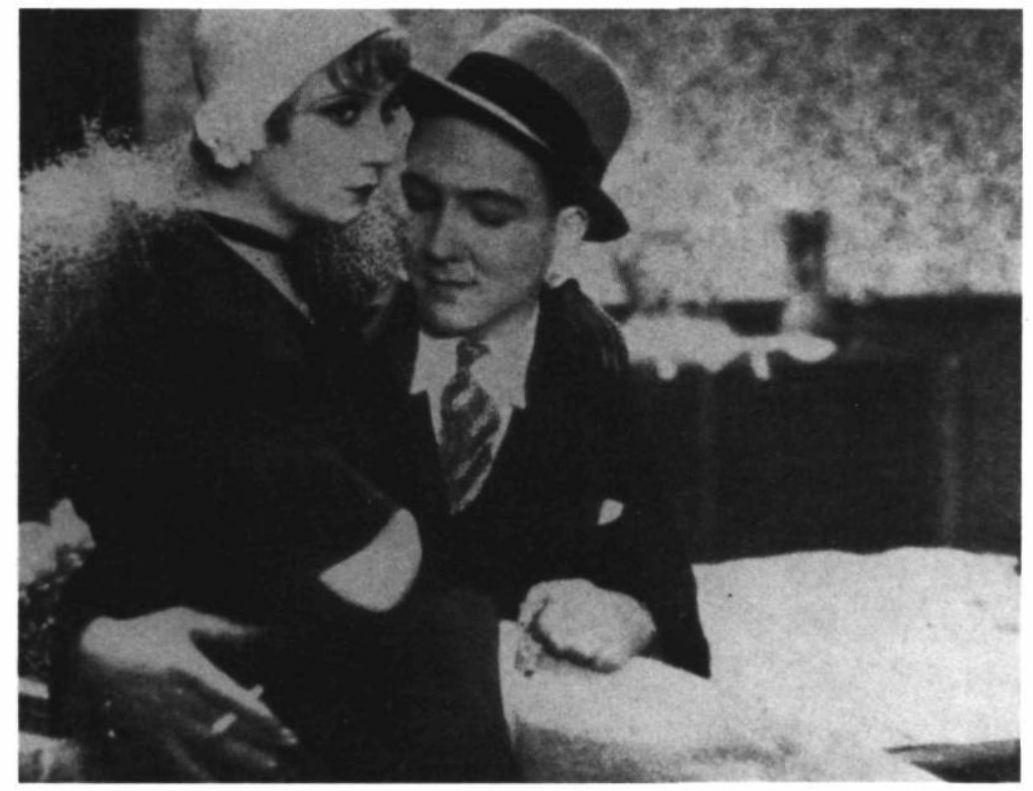

La Chienne de Jean Renoir (1931)

Coll. Cinémathèque québécoise 
Cette «soustraction» est dite visuellement par la manière dont un court fragment est détaché de l'ensemble par deux fermetures au noir qui l'enserrent. À l'intérieur même du cristal qui coïncide avec la chanson, ce fragment constitue un autre cristal, plus limpide que le premier. La caméra amorce un mouvement ascendant depuis le groupe des badauds et des musiciens dans la rue, elle monte le long de la façade jusqu'au troisième étage: survient alors une fermeture au noir. Au plan suivant apparaît un chaton noir qui se lèche. La caméra révèle par un travelling avant et un panoramique vertical trois pots de géranium et une fenêtre ouverte qui donne sur la chambre. Le corps de la jeune femme est étendu transversalement sur le lit, le buste à la renverse; Legrand, à genoux sur le sol, lui embrasse les mains : l'oreiller est éclaboussé de sang, comme le zinc à côté du chaton est éclaboussé d'eau. Le plan suivant montre la rue alors que le chanteur entame un nouveau couplet. Legrand et Lulu réapparaissent dans le troisième plan, cadré depuis la fenêtre et s'achevant par une fermeture au noir. Dans ce passage, les paroles de la complainte, l'espace d'un instant, semblent en harmonie avec les images. Lors du mouvement de la caméra le long de la façade, on entend: «Devant moi, devant moi, ma belle inconnue...»; or, le plan qui vient après est celui où Legrand est agenouillé auprès du visage de Lulu. Ensuite, la mention de la fenêtre dans la chanson coïncide avec le cadrage depuis ce lieu.

Ce moment d'harmonie ne dure pas. Pendant la seconde fermeture au noir, une ellipse se produit dans la chanson qui peut correspondre au temps nécessaire à Legrand pour se rhabiller et descendre les étages. Et quand Legrand a quitté la scène, la ritournelle (musique et paroles) disparaît sur le plan de la voiture de Dédé roulant dans une rue (écart unique dans ce paysage puisqu'il montre un espace autre que ceux de la rue et de l'appartement). Elle reprend au plan suivant, seulement interprétée par les musiciens et elle se poursuit sans discontinuer, le temps singulièrement court que met Dédé à monter les étages, à découvrir le corps, puis à redescendre. On peut parler à propos de ce personnage de «durée fatale» et d'accélération. Soit la ritournelle s'accélère en raison d'ellipses, soit c'est le mouvement même du personnage qui est accéléré.

Ce passage présente bien un «cristal» d'espace-temps. En effet, si grâce à la chanson des rues plusieurs moments se trouvent rassemblés, grâce à elle également des espaces sont mis en relation: un intérieur et un extérieur, un haut et un bas. Des jeux de mots élémentaires définissent la situation et renforcent l'opposition : à l'étage, Legrand tombe de haut tandis que dans la rue (très en pente) Dédé glisse sur une pente fatale le conduisant tout droit à la 
guillotine. Cependant, les croisements qui se produisent d'un lieu à un autre sont l'un des traits caractéristiques de la scène. L'intérieur qui devrait être clos est largement ouvert : sur deux de ses parois des portes sont ouvertes et sur la troisième une fenêtre l'est également, alors que la forme close de la chanson appartient à l'espace extérieur. Dans cette perspective, un détail rapporté par Renoir renforce l'idée de chiasme :

On a mis en bas, dans la rue, le son qui était pris dans la chambre, alors que j'avais un son pris dans la rue, plein de cornes d'autos, de bruits magnifiques, qui a été perdu (p. 53).

La substitution que regrette le réalisateur s'intègre bien à la série des échanges entre les deux espaces.

Dans La Bête humaine, l'idée est reprise d'un meurtre accompli tandis que non loin s'égrènent les paroles d'une chanson populaire, Le p'tit coeur de Ninon. L'effet obtenu est différent, non seulement parce que les personnages du drame ne peuvent entendre cette chanson, mais surtout parce l'entrecroisement des lieux et des temps est inexistant dans le film de 1938 : les paroles servent seulement de contrepoint à l'action principale et disent le climat de sentimentalité naïve dans lequel les personnages ont vécu leur aventure. L'assassinat de l'être aimé se déroule aussi de façon différente dans les deux films : la mort de Lulu résulte d'une pulsion criminelle de Legrand qui trouve à se justifier dans la découverte atterrante pour le personnage du fait qu'il a été trompé tout au long, et elle a pour conséquence une dérive vers la clochardisation qui fait de Legrand un chien; la mort de Séverine, elle, n'a aucune autre justification que la double nature de Lantier, donné d'emblée comme une bête humaine par la faute de l'hérédité: son suicide est la suite obligée du meurtre qu'il commet et de sa lucidité retrouvée. L'action de La Bête humaine relève de la fatalité inéluctable, alors que La Chienne n'est ni un drame ni une comédie: Renoir se sent plus à l'aise dans la satire ambiguë que dans la clarté tragique. L'utilisation qu'il fait de la ritournelle s'en trouve bridée dans La Bête humaine, où ne s'exprime que la puissance de la mort, alors que dans La Chienne, la force de vie est présente jusque dans la scène du meurtre. 


\section{OUVRAGES CITÉS}

Chion, Michel. Le Son au cinéma. Paris : Cahiers du cinéma / éd. de l'Étoile, 1985.

Deleuze, Gilles. Cinéma 2. L'Imagc-temps. Paris : Minuit, 1985.

Deleuze, Gilles et Félix Guattari. Mille plateaux. Paris : Minuit, 1980.

Renoir, Jean. Jean Renoir, Entretiens et Propos. Paris : Cahiers du cinéma / éd. de l'Étoile, 1979.

Schefer, Jean-Louis. L'Homme ordinaire du cinéma. Paris : Cahiers du cinéma / Gallimard, 1980. 